\title{
AVALIAÇÃO DO TAMANHO DE AEROPORTOS PORTUGUESES COM RELAÇÕES MULTICRITÉRIO DE SUPERAÇÃO
}

\author{
João Carlos Correia Baptista Soares de Mello * \\ Dep. Eng. Produção / Univ. Federal Fluminense (UFF) \\ Niterói - RJ \\ jcsmello@producao.uff.br
}

Eliane Gonçalves Gomes

Embrapa

Brasília - DF

eliane.gomes@embrapa.br

Luiz Flávio Autran Monteiro Gomes

Faculdades Ibmec/RJ - Ibmec Business School

Rio de Janeiro - RJ

autran@ibmecrj.br

Luiz Biondi Neto

Dep. Eng. Eletr. e Telecom./ Univ. Est. Rio de Janeiro (UERJ)

Rio de Janeiro - RJ

lbiondi@uerj.br

\section{Lidia Angulo Meza}

Dep. Ciência dos Materiais / Univ. Federal Fluminense (UFF)

Volta Redonda - RJ

lidia_a_meza@yahoo.com

* Corresponding author / autor para quem as correspondências devem ser encaminhadas

Recebido em 11/2003; aceito em 07/2005 após 2 revisões

Received November 2003; accepted July 2005 after 2 revisions

\section{Resumo}

Nos estudos que envolvem aeroportos é recomendável que estes sejam divididos em categorias, de acordo com seu tamanho. Apesar de essa divisão ser um problema de análise multicritério, os dados são disponibilizados de forma desagregada, ou seja, cada aeroporto é avaliado em cada critério de tamanho de forma isolada. Este artigo apresenta uma variante de um método multicritério da Escola Francesa, nomeadamente o ELECTRE I, para avaliar o tamanho de nove aeroportos portugueses e agrupá-los por categorias de tamanho. Os critérios utilizados referem-se à movimentação de aeronaves, à movimentação de passageiros e à movimentação de cargas. Os resultados obtidos são comparados com outros dois modelos baseados em soma ponderada e análise de envoltória de dados.

Palavras-chave: alocação em classes; ELECTRE; intransitividade; aeroportos.

\begin{abstract}
In airport studies it is quite common to define categories of airports according to their sizes. Regarding size, each airport is usually classified according to only one criterion, although the problem of determining the airport size is clearly a multicriteria one. This paper employs a new approach for classifying airports according to their sizes by making use of a variant of the multicriteria method ELECTRE I. The new approach is applied to evaluate the size of Portuguese airports and to allocate these airport to well-defined size categories. The amount of cargo and the number of passengers, as well as number of aircraft landing in the airport, are the used criteria. The obtained results are compared with two additional models based on weighted sum and data envelopment analysis.
\end{abstract}

Keywords: class division; ELECTRE; intransitivity; airports. 


\section{Introdução}

A International Civil Aviation Organization (ICAO) recomenda, em qualquer estudo de aeroportos, que eles sejam divididos em categorias de acordo com seu tamanho (Palhares, 2001). A divisão por tamanho é, claramente, um problema de análise multicritério. É corrente usar diversos critérios para a ordenar os aeroportos por tamanho, tais como movimentação de passageiros, movimentação de aeronaves, movimentação de carga e área ocupada, sem, no entanto, usá-los conjuntamente.

Embora existam diversos métodos para avaliar aeroportos de diferentes tamanhos de forma agregada, estes referem-se apenas a estudos de eficiência, como os apresentados por Soares de Mello \& Gomes (2004) e por Fernandes \& Pacheco (2002). A divisão em categorias é fundamental para estudos de impacto econômico e priorização de ações administrativas, entre outros (Palhares, 2001).

Este artigo apresenta um método multicritério da Escola Francesa para avaliar o tamanho dos aeroportos portugueses. Nesta avaliação, pretende-se agrupar os aeroportos em classes e ordenar as classes, sem necessariamente ordenar os aeroportos dentro das classes. Dois motivos levaram à escolha desses aeroportos. O primeiro é o fato de serem relativamente poucos (nove), o que permite um estudo completo sem a necessidade de implementar algoritmos complexos, nem programas computacionais.

O segundo motivo é a dificuldade em obterem-se dados completos relativos aos aeroportos brasileiros. Este é um problema crônico do setor, discutido em Espírito Santo Jr. (2000). Os dados disponíveis para o caso brasileiro apenas referem-se aos vinte aeroportos melhor colocados em cada critério. Em muitos casos, um aeroporto que aparece bem colocado em um critério, não consta da lista dos maiores em outro critério. Por exemplo, os aeroportos de Macaé (no Estado do Rio de Janeiro) e Campo de Marte (na Cidade de São Paulo) estão entre os vinte maiores em número de decolagens (grande movimentação de helicópteros), mas têm baixa movimentação de carga e passageiros. Essa situação dificulta o uso correto de técnicas multicritério.

\section{Aeroportos Avaliados}

Foram estudados os aeroportos administrados pela ANA (Aeroportos e Navegação Aérea) e pela ANA Madeira. A seguir faz-se uma breve descrição desses aeroportos. Nessa lista não estão incluídos nem os pequenos aeroportos regionais, nem um importante aeroporto da Ilha Terceira. Este fica localizado na Base Aérea das Lages, importante ponto estratégico administrado pelos Estados Unidos.

\subsection{Aeroporto da Portela de Sacavém (Aeroporto de Lisboa)}

É o principal aeroporto português e contribui de forma expressiva para o desenvolvimento econômico da cidade e de toda a região de Lisboa.

Dele partem diversos vôos diários para outras cidades portuguesas e outros países europeus, para África e as Américas do Norte e do Sul.

É o aeroporto europeu de onde parte o maior número de vôos para destinos no Brasil, e a adequação às necessidades geradas pelo aumento de tráfego tem originado um progressivo 
investimento na sua modernização. Há ainda um constante debate sobre a necessidade de um novo aeroporto e sua localização.

\subsection{Aeroporto Francisco Sá Carneiro (Aeroporto do Porto)}

É o aeroporto que serve a toda região Norte de Portugal, marcada por crescente industrialização e localizado a cerca de $11 \mathrm{Km}$ da cidade do Porto.

Até há alguns anos, os vôos que serviam esse aeroporto faziam, em sua maioria, uma escala prévia no aeroporto de Lisboa. Atualmente, o número de vôos dedicados a esse aeroporto está em expansão.

\subsection{Aeroporto de Faro}

É um aeroporto dedicado essencialmente à região turística do Algarve e está localizado a aproximadamente $4 \mathrm{~km}$ da capital Algarvia. A maioria dos vôos que servem a esse aeroporto parte para destinos europeus, além de uma grande freqüência de vôos charter.

\subsection{Aeroporto João Paulo II (Aeroporto de Ponta Delgada)}

O aeroporto João Paulo II localiza-se em Ponta Delgada, cidade mais populosa da região autônoma dos Açores, na ilha de São Miguel. Dele partem essencialmente vôos para outras cidades portuguesas e para os Estados Unidos. Quase todos os vôos são operados pelo Grupo SATA (Sociedade Açoriana de Transporte Aéreo).

\subsection{Aeroporto de Santa Maria}

Foi o aeroporto pioneiro do Arquipélago dos Açores com 50 anos de certificação e abertura à Aviação Civil. Está localizado na ilha de mesmo nome e próximo ao seu principal centro populacional. É hoje um aeroporto essencialmente vocacionado para escalas técnicas e vôos dentro da região dos Açores.

\subsection{Aeroporto da Horta}

O aeroporto da Horta, localizado na ilha de Faial, é o mais importante em todo o grupo central do arquipélago dos Açores (São Jorge, Pico e Faial). Esse aeroporto garante não só as ligações com a parte continental de Portugal, mas também com as outras ilhas do Arquipélago. É, assim, pólo dinamizador da economia da região.

\subsection{Aeroporto das Flores}

Serve à cidade de Santa Cruz das Flores, localizada no grupo ocidental do Arquipélago dos Açores, na ilha das Flores. Tem essencialmente tráfego regional.

\subsection{Aeroporto Internacional da Madeira}

Localizado na Ilha da Madeira, serve à cidade de Funchal. Tem grande movimento turístico proveniente de toda a Europa e também importante movimento de carga para abastecimento de todo o arquipélago da Madeira. 
Durante muitos anos foi um aeroporto extremamente perigoso, no qual só podiam operar aviões de pequeno e médio porte. Recentemente, passou por um processo de modernização e ampliação e, atualmente, nele pode operar qualquer tipo de avião. Uma das características curiosas deste aeroporto é que uma grande parte da pista é uma estrutura elevada sobre o mar.

\subsection{Aeroporto do Porto Santo}

Localizado na Ilha de Porto Santo, a menor das duas ilhas habitadas do arquipélago da Madeira, este aeroporto servia de base para os vôos intercontinentais, antes da ampliação do Aeroporto Internacional da Madeira.

Atualmente, os vôos que partem de Porto Santo são, em sua maioria, operados por aviões hélice de pequeno porte, com destino à Ilha da Madeira.

\section{Apoio Multicritério à Decisão}

O Apoio Multicritério à Decisão (AMD) pode ser definido como o conjunto de técnicas que têm a finalidade de investigar um número de alternativas, sob múltiplos critérios e objetivos em conflito. É possível gerar soluções compromisso e uma hierarquização das alternativas, de acordo com o grau de atração destas para o tomador de decisão (Gomes et al., 2002, 2004).

Segundo Bouyssou (1990), uma abordagem multicritério apresenta as seguintes vantagens:

- torna viável a construção de uma base para o diálogo entre analistas e decisores, que fazem uso de diversos pontos de vista comuns;

- provê facilidade em incorporar incertezas aos dados sobre cada ponto de vista;

- permite encarar cada alternativa como um compromisso entre objetivos em conflito. Este argumento destaca o fato de que raramente será encontrada uma situação em que exista uma alternativa superior às restantes sobre todos os pontos de vista.

Em um problema multicritério é necessário, em primeiro lugar, estabelecer claramente qual o objetivo da análise. Classicamente podem ser definidas três problemáticas multicritério: ordenação ( $\mathrm{P} \gamma)$, escolha $(\mathrm{P} \alpha)$ e alocação em classes $(\mathrm{P} \beta)$ (Gomes et al., 2004). Este artigo não trata da problemática $\mathrm{P} \alpha$ e pretende concentrar-se numa variante da $\mathrm{P} \beta$, embora em alguns pontos faça uso da $\mathrm{P} \gamma$. Esta consiste em obter uma ordenação total das alternativas, da pior para a melhor ou vice-versa. Para tanto, deve-se estabelecer uma relação de pré-ordem no conjunto das alternativas, ou seja, uma relação binária que seja reflexiva, transitiva e total (Barba-Romero \& Pomerol, 1997).

A problemática $\mathrm{P} \beta$ consiste em agrupar as alternativas em classes supostamente de características semelhantes. Segundo Roy (1985), cabe ao decisor estabelecer uma parametrização que permita essa divisão. Em Álgebra é feita uma definição mais abrangente, que consiste em estabelecer no conjunto das alternativas uma relação de equivalência, ou seja, uma relação binária que seja reflexiva, simétrica e transitiva (Domingues \& Iezzi, 1976). Esta definição é a adotada neste artigo e, para evitar conflitos de nomenclatura, será denominada "pseudo divisão em classes" ou $\mathrm{P} \beta$ '.

Segundo Dias (2002), a P $\beta$ pode ser subdividida em alocação nominal ou ordinal. Esta subdivisão pode ser estendida à $\mathrm{P} \beta$ '. A primeira divide as alternativas em classes, sem 
estabelecer hierarquia alguma entre elas. Já a alocação ordinal, após a divisão em classes, hierarquiza estas, ou seja, é feita uma P $\gamma$ em que as novas alternativas são as classes.

Na modelagem de um problema multicritério deve-se ainda definir as alternativas, o método a ser usado, os critérios e quem atua como decisor (Soares de Mello et al., 2003a).

Historicamente, os primeiros métodos da chamada Escola Francesa do AMD foram os métodos ELECTRE I e II (Roy, 1968; Roy \& Bertier, 1971, 1973; Rogers et al., 2000). Esses métodos definem uma série de processos sobre as ações consideradas, pertencentes ao conjunto de possíveis soluções para o problema de decisão analisado. Inicialmente, essas ações são dispostas em uma tabela cruzada com os vários critérios selecionados, formando uma matriz de custos, e onde são atribuídos pesos para os vários critérios. Embora não se pretenda aqui explicar esses dois métodos multicritério pioneiros, uma vez que os mesmos acham-se sobremaneira detalhados na literatura especializada (Roy \& Bouyssou, 1993; Gomes et al., 2002, 2004), os elementos mais importantes do primeiro deles serão apresentados na seção 3.1.

Neste artigo estuda-se o tamanho dos aeroportos, por meio de destilações ascendentes e descendentes obtidas dos grafos representativos das relações de superação fornecidas por uma variante do método ELECTRE I proposta neste artigo. Quando não existem ciclos de intransitividade, essas destilações induzem uma pré-ordem no conjunto de aeroportos, ou seja, resolvem uma problemática $\mathrm{P} \gamma$. Quando os ciclos existem, o ELECTRE I clássico resolve uma $\mathrm{P} \alpha$, e a variante proposta uma $\mathrm{P} \beta$ ordinal.

\subsection{Método ELECTRE I e a variante proposta}

Todos os métodos da família ELECTRE são baseados em relações de superação. Ao contrário dos métodos da Escola Americana, não fornecem um indicador final agregado, resultado de um critério de síntese. O seu uso neste trabalho decorre de alguns fatores. Em primeiro lugar não são compensatórios, isto é, não permitem que um mau desempenho em algum critério seja compensado por um excelente desempenho em outro.

O método ELECTRE I, o primeiro de uma família de métodos que usam o conceito de superação, tenta resolver a problemática conhecida como $\mathrm{P} \alpha$, isto é, tenta esclarecer a decisão por meio da escolha de um subconjunto, tão restrito quanto possível, contendo as ações que foram consideradas como melhores. Para atingir esse objetivo, a exploração das relações de superação deverá ser conduzida de tal modo a obter-se um subconjunto $K$ de alternativas possíveis, também chamado de mínimo subconjunto dominante por conter as seguintes propriedades:

- uma alternativa pertencente ao subconjunto $K$ não é superada por nenhuma outra alternativa também pertencente a $K$;

- para toda alternativa não pertencente ao subconjunto $K$, existe uma alternativa pertencente a $K$ que a supera.

Por conseguinte, pode-se afirmar que o método ELECTRE I, em sua tentativa de solucionar uma dada problemática $\mathrm{P} \alpha$, consiste em encontrar o mínimo conjunto de alternativas não dominadas, que seria o núcleo do grafo reduzido gerado pelas relações de preferência construídas a partir dos julgamentos do(s) agente(s) de decisão.

Já o método ELECTRE II tem como objetivo a solução da problemática P $\gamma$. Este método usa duas relações de superação, mas pelo fato de trabalhar somente com critérios verdadeiros, 
tem sido preterido pelos métodos ELECTRE III e IV (Dias et al., 1996). Aplicações desses podem ser vistas em Miranda \& Almeida (2004) e Gomes \& Rocha (1999).

Existem duas versões do método ELECTRE I, designadas por ELECTRE Iv e ELECTRE Is. A primeira substitui a exigência de um limiar de discordância por um limiar de veto, considerado por alguns autores (Dias et al., 1996) como mais fácil de apresentar ao decisor. A segunda versão tem a vantagem de trabalhar com pseudo critérios e, portanto, incorporar aos resultados incerteza ou imprecisão associada aos desempenhos. Para tal, exige a definição de um limiar de veto e de um limiar de preferência estrita (idênticos ou não). A filosofia do método ELECTRE Is poderá ser útil nos desenvolvimentos futuros da variante proposta neste artigo, no sentido de não considerar um aeroporto preferível a outro quando as diferenças, que podem resultar de imprecisão dos dados, forem bastante pequenas.

Um outro método desta família é o ELECTRE TRI (Roy, 1985; Yu \& Roy, 1992), que por meio de relações difusas resolve a $\mathrm{P} \beta$, desde que o decisor forneça certos parâmetros de classificação. Exemplos de aplicação do método ELECTRE TRI são encontrados em Miranda \& Almeida (2003) e Costa et al. (2004).

O conceito fundamental no uso do método ELECTRE I é a relação de preferência fraca: dizse que, em relação a um determinado critério, a alternativa $A$ tem preferência fraca sobre a alternativa $B$ se o decisor está em dúvida se $A$ é realmente preferível a $B$ ou se são indiferentes uma a outra. Em outras palavras, $A$ tem preferência fraca sobre $B$ se $A$ não é pior que $B$ em determinado critério. Pode ainda acontecer o seguinte: $A$ prefere fracamente $B$ e, inversamente, $B$ também tem preferência fraca sobre $A$. Caso isso aconteça, fica caracterizada a indiferença entre as duas alternativas no critério analisado. Se, em um determinado critério, $A$ prefere $B$ (sendo esta preferência estrita ou fraca), então esse critério faz parte da chamada coalizão concordante em relação a $A$ superar $B$. O método ELECTRE I considera ainda critérios de discordância, que formam uma coalizão discordante, o que não será empregado neste artigo, devido à dificuldade de interpretar os limiares de discordância.

Após a análise par a par de todas as alternativas segundo todos os critérios, é necessário construir a relação de superação. Diz-se que $A$ supera $B$ se existe uma relação de preferência fraca de $A$ para $B$ em um número pré-definido de critérios. Este número define a configuração do método e pode ser escolhido de forma a favorecer uma perfeita ordenação final (se as opiniões dos decisores forem firmes) ou, pelo contrário, não possibilitar uma ordenação total (quando as opiniões dos decisores não são tão seguras).

As dificuldades em obter-se uma ordenação total devem-se aos ciclos de intransitividade. Estes ocorrem quando a alternativa $A$ supera $B, B$ supera $C$, que, por sua vez, supera $A$. Uma situação destas impossibilita uma ordenação entre $A, B$ e $C$. Embora estes ciclos sejam o grande entrave ao uso dos métodos ELECTRE, neste artigo será tirado partido deles. Normalmente o método usado para divisão em classes é o ELECTRE TRI, que usa conceitos de lógica difusa (Roy \& Bouyssou, 1993; Gomes et al., 2004). No entanto, de forma mais simples, pode-se considerar a pseudo divisão em classes correspondendo aos ciclos de intransitividade e, assim, usa-se uma aparente desvantagem do método para obter informação relevante.

Para formalizar esta variante do método ELECTRE I aqui proposta, devem ser definidas duas novas relações. A primeira é uma relação binária pseudo-reflexiva em um subconjunto das alternativas, que é uma relação cujo grafo representativo admite um caminho de qualquer alternativa para ela própria, ou seja, existem ciclos começando em todas as alternativas deste subconjunto. A segunda relação a ser definida é uma relação pseudo-simétrica da seguinte 
forma: dadas duas alternativas $A$ e $B$, pertencentes ao mesmo subconjunto, sempre existirá um caminho de A para $\mathrm{B}$ e outro caminho da alternativa $B$ para a alternativa $A$. Todos os caminhos mencionados nestas duas definições só podem conter alternativas do subconjunto considerado. A uma relação binária que no subconjunto considerado seja pseudo-reflexiva e pseudo-simétrica chamar-se-á de relação de pseudo-equivalência.

Assim como no caso da existência de relações de equivalência, a divisão em classes é feita pela partição induzida pelas classes de equivalência. No método aqui proposto, os subconjuntos disjuntos maximais das alternativas, nos quais seja possível definir relações de pseudo-equivalência, formam as classes pretendidas. A definição de uma relação pseudoreflexiva garante que existem ciclos de intransitividade nas classes e, portanto, esta definição formal equivale à definição informal anteriormente colocada.

Os resultados obtidos independem de definições de limites por parte dos decisores. A atuação dos decisores ocorre na escolha dos critérios e na qualificação de maioria para existir a relação de superação.

Assim, a principal diferença entre a variante proposta e descrita nos parágrafos anteriores e o método ELECTRE I clássico está na fase de exploração do grafo. Enquanto o método original contorna o problema dos ciclos de intransitividade ao determinar o núcleo do grafo (Boaventura Netto, 2002) e concentra-se no problema da escolha de uma alternativa, a variante usada neste artigo usa toda a informação contida no grafo. Quando é possível (caso inexistam intransitividades), a variante estabelece uma relação de ordem. Quando existem intransitividades, faz uma alocação em classes com uso das relações de pseudo-equivalência. Dessa forma, a variante proposta ora resolve a problemática $\mathrm{P} \gamma$, ora a problemática $\mathrm{P} \beta$ ordinal, dependendo do caso em estudo e da exigência do número de critérios na coligação concordante.

\section{Modelagem}

Conforme apresentado anteriormente, em modelagem multicritério devem-se definir alternativas, critérios, método e decisores. As alternativas em avaliação são os nove aeroportos portugueses operados pela ANA e pela ANA Madeira, cuja descrição está na seção 2.

Os critérios usados devem formar uma família coerente. Foram considerados os seguintes critérios: movimentação de aeronaves (medido pelo número de decolagens), movimentação de passageiros (medido em quantidade de passageiros embarcados) e movimentação de carga (medido em toneladas). Para o que se pretende avaliar, esta família é exaustiva, já que estão relacionados todos os critérios que se referem à importância econômica do aeroporto. Poderse-ia alegar que existe falta de exaustividade por não ter sido considerado o critério área ocupada. A decisão de não incluir este atributo justifica-se por este parâmetro representar, essencialmente, tamanho físico do aeroporto e não estar diretamente relacionado à sua movimentação e importância econômica.

Por outro lado, os critérios não são redundantes, já que a retirada de um deles retira a exaustividade da família. Mais uma vez, pode ser alegado que existe uma certa redundância, já que movimentação de passageiros e carga provoca maior movimentação de aeronaves. No entanto, esta argumentação não contraria a definição de não redundância de Roy (Roy \& Bouyssou, 1993); apenas garante que os critérios não são independentes. Esta constatação impede o uso de métodos como a MAUT aditiva (Gomes et al., 2004).

Finalmente, a coesão da família de critérios escolhida é garantida na quantificação dos critérios. 
Os dados foram obtidos (ANA, 2002) e referem-se ao período de janeiro a setembro de 2002. A Tabela 1 apresenta os valores de cada alternativa em cada critério.

Atuou como decisor um dos autores, com razoável conhecimento de aeroportos e transporte aéreo.

O método a ser usado é a variante do método ELECTRE I proposta neste artigo e descrita no item 3.1. Em uma primeira configuração considerou-se que a superação exigia a maioria simples, ou seja, um aeroporto é maior que outro se tiver maior movimentação em pelo menos dois dos critérios considerados.

Foi assumida, inicialmente, igual importância para os dois fatores geradores de movimento (passageiros e carga) bem como entre cada um destes em relação ao movimento gerado. $\mathrm{Ou}$ seja, foram atribuídos pesos iguais a todos os critérios. Como o decisor é um dos autores, ao invés de uma autoridade aeroportuária, será feita uma análise de sensibilidade da variação dos pesos, ou seja, será verificado o que aconteceria com um decisor com opiniões diferentes.

Tabela 1 - Dados de alternativas e critérios.

\begin{tabular}{cccc}
\hline \multirow{2}{*}{ Alternativas } & \multicolumn{3}{c}{ Critérios } \\
\cline { 2 - 4 } & $\begin{array}{c}\text { Movimentação de } \\
\text { aeronaves }\end{array}$ & $\begin{array}{c}\text { Movimentação de } \\
\text { passageiros }\end{array}$ & $\begin{array}{c}\text { Movimentação de } \\
\text { carga }\end{array}$ \\
\hline Lisboa & 92.856 & 8.118 .068 & 65.736 \\
Porto & 34.442 & 2.279 .413 & 28.719 \\
Faro & 28.386 & 4.400 .454 & 1.415 \\
Ponta Delgada & 8.412 & 686.989 & 5.982 \\
Santa Maria & 1.159 & 60.100 & 175 \\
Horta & 3.557 & 170.522 & 855 \\
Flores & 888 & 27.455 & 180 \\
Internacional da Madeira & 20.248 & 1.924 .857 & 7.125 \\
Porto Santo & 5.034 & 158.784 & 183 \\
\hline
\end{tabular}

Devido à inexistência de relações de discordância e à igualdade de pesos, esta configuração fornece as mesmas relações de superação do método de Condorcet, como mostrado em Soares de Mello et al. (2003c) e é, portanto, um método ordinal.

Na segunda configuração, a superação ocorre com a preferência fraca em apenas um critério. Naturalmente, esta configuração favorece o surgimento de indiferenças e intransitividades. Nesta configuração, um aeroporto só não é superado por outro se houver unanimidade de critérios em favor do segundo. Tal fato torna freqüente que um aeroporto, ao mesmo tempo, supere e seja superado por outro.

\section{Resultados}

Com a primeira configuração do método ELECTRE I obtém-se um grafo de superação, cuja matriz de adjacência (Boaventura Netto, 2002) está apresentada na Tabela 2. 
Nesse grafo não existem ciclos de intransitividade. Portanto, o método conduz a uma ordenação total. Essa situação não é usual na aplicação dos métodos ELECTRE e ocorreu devido a uma grande semelhança entre as ordenações promovidas pelos três critérios e ao fato de essa configuração do ELECTRE I representar uma situação em que o decisor tem seus julgamentos bem fundamentados. A Tabela 3 mostra essa ordenação.

Tabela 2 - Matriz de adjacência da primeira configuração do método.

\begin{tabular}{|c|c|c|c|c|c|c|c|c|c|}
\cline { 2 - 9 } \multicolumn{1}{c|}{} & Lisboa & Porto & Faro & $\begin{array}{c}\text { Ponta } \\
\text { Delgada }\end{array}$ & $\begin{array}{c}\text { Santa } \\
\text { Maria }\end{array}$ & Horta & Flores & $\begin{array}{c}\text { Int. da } \\
\text { Madeira }\end{array}$ & $\begin{array}{c}\text { Porto } \\
\text { Santo }\end{array}$ \\
\hline Lisboa & & 1 & 1 & 1 & 1 & 1 & 1 & 1 & 1 \\
\hline Porto & & & 1 & 1 & 1 & 1 & 1 & 1 & 1 \\
\hline Faro & & & & 1 & 1 & 1 & 1 & 1 & 1 \\
\hline Ponta Delgada & & & & 1 & 1 & 1 & & 1 \\
\hline Santa Maria & & & & & & & 1 & & \\
\hline Horta & & & & & 1 & & 1 & & 1 \\
\hline Flores & & & & & & & & & \\
\hline Int. da Madeira & & & 1 & 1 & 1 & 1 & & 1 \\
\hline Porto Santo & & & & & 1 & & 1 & & \\
\hline
\end{tabular}

Tabela 3 - Ordenação resultante da primeira configuração do ELECTRE I.

\begin{tabular}{cc}
\hline Posição & Alternativa \\
\hline 1 & Lisboa \\
2 & Porto \\
3 & Faro \\
4 & Internacional da Madeira \\
5 & Ponta Delgada \\
6 & Horta \\
7 & Porto Santo \\
8 & Santa Maria \\
9 & Flores \\
\hline
\end{tabular}

Como o decisor foi um dos autores, e não uma autoridade aeroportuária, é conveniente investigar quais seriam as mudanças nos resultados caso houvesse um decisor com outras opiniões. Para tal, é apresentada em seguida uma análise de sensibilidade para a variação dos pesos. Em uma primeira situação, atribui-se peso $n>1$ ao critério movimentação de aeronaves, o que significa aumentar a importância do movimento gerado em relação aos fatores geradores desse movimento. Neste caso, a única alteração na ordenação mostrada na Tabela 3 ocorre para $n>2$ e é a inversão entre Horta e Porto Santo.

Para a situação inversa (fatores geradores mais importantes que o movimento gerado), atribuindo-se o peso $n>1$ aos critérios movimentação de passageiros e movimentação de carga, não houve alteração na ordenação da Tabela 3 para qualquer valor de $n$. Não foi feita análise de pesos diferenciados para carga e passageiros. Uma análise deste tipo exigiria a 
possibilidade de atribuir pesos diferentes conforme o aeroporto analisado, já que cada um apresenta suas próprias especificidades. Análises deste tipo podem ser conduzidas, por exemplo, com Análise de Envoltória de Dados (DEA), como mostrado em Fernandes \& Pacheco (2002) e Soares de Mello \& Gomes (2004).

Esta análise de sensibilidade mostrou uma pequena variação de resultados com a alteração dos pesos, o que indica que a ordenação obtida é pouco dependente da opinião do decisor. Seria ainda possível fazer uma análise para o caso de movimento de passageiros e carga terem pesos diferentes. Esta análise tem pouco sentido na avaliação global do tamanho do aeroporto. Seria interessante fazê-la se o objetivo fosse avaliar a vocação do aeroporto como industrial ou de turismo. Aeroportos que tivessem melhor classificação quando fosse atribuído peso maior ao movimento de passageiros seriam mais voltados ao turismo, caso contrário estariam mais próximos da situação de um aeroporto industrial. No entanto, o objetivo deste artigo é penas avaliar o tamanho, o que torna desnecessária esta segunda análise de sensibilidade.

Os resultados obtidos desta primeira configuração conduziram a uma $\mathrm{P} \gamma$, o que não era $o$ objetivo do estudo. Na verdade, pode-se considerar que a $\mathrm{P} \gamma$ gerada é uma $\mathrm{P} \beta$ ' com número de alternativas igual ao número de classes. No entanto, esta é uma $\mathrm{P} \beta$ ' de pouca utilidade. Para obter uma $\mathrm{P} \beta$ ' no sentido estrito do termo, devem-se relaxar as opiniões do decisor, de modo a favorecer o surgimento de indiferenças e intransitividades. Esse relaxamento é feito exigindo-se unanimidade de critérios para que um aeroporto não seja superado por outro.

Na segunda configuração apenas o aeroporto de Lisboa não é superado por algum outro e pode ser considerado como componente único de uma primeira classe. Em seguida, surge um conjunto de 4 aeroportos (Funchal, Ponta Delgada, Faro e Porto) em que o grafo da relação de superação admite caminhos entre qualquer um de seus componentes. Além disso, estes aeroportos não são superados por algum outro fora de seu conjunto, exceto por Lisboa, formando, assim, uma segunda classe. Analogamente, os aeroportos de Horta e Porto Santo formam a terceira classe, e Flores e Santa Maria constituem a última classe. Os grafos que representam as relações de superação dentro das classes são mostrados na Figura 1.

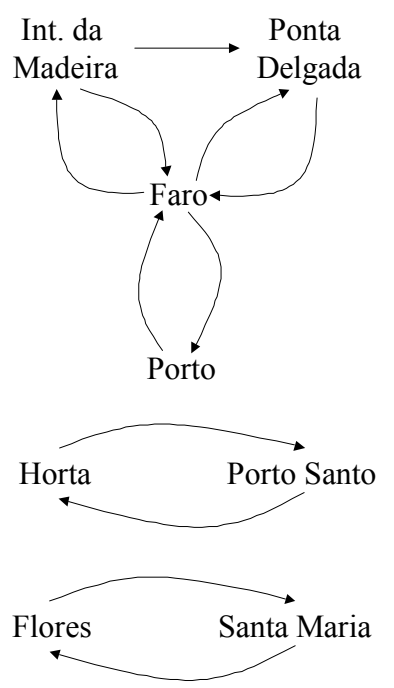

Figura 1 - Ciclos de intransitividade da segunda configuração do ELECTRE I. 
Essa configuração reflete menor grau de certeza por parte do decisor ao emitir seus julgamentos e, como conseqüência, surgem os ciclos de intransitividade. Embora seja impossível fazer uma ordenação total, a existência de ciclos conduz a uma pseudo divisão em classes, apresentada na Tabela 4.

Tabela 4 - Classes dos aeroportos de acordo com a segunda configuração.

\begin{tabular}{cc}
\hline Classe & Aeroportos \\
\hline I & Lisboa \\
II & Porto, Ponta Delgada, Faro, Internacional da Madeira \\
III & Horta, Porto Santo \\
IV & Flores, Santa Maria \\
\hline
\end{tabular}

\section{Comparação com Outros Métodos}

Embora o foco deste artigo seja a pseudo divisão em classes baseada em relações de superação, é interessante verificar se seria possível obter resultados semelhantes com outros métodos multicritério. Para esta comparação foram escolhidos métodos com base em soma ponderada, visto serem estes os mais difundidos.

Duas abordagens podem ser usadas. A primeira consiste em avaliar a atratividade de alternativas e critérios, com a conseqüente atribuição de pesos aos critérios e sua agregação em uma soma ponderada. Um dos métodos que permitiria esta análise é o MACBETH (Bana e Costa \& Vansnick, 1994), com aplicações exemplificadas em Bana e Costa et al. (2002) e Soares de Mello et al. (2003b), entre outros. O uso deste método exigira a definição de limiares para as classes, ou seja, fazer uma $\mathrm{P} \beta$ em sentido estrito.

Como neste artigo o objetivo é deixar que as alternativas se auto agrupem, sem a definição prévia de limiares ou parâmetros, foram formulados dois modelos alternativos. O primeiro deles atribui o mesmo esquema de pesos para todas as alternativas, sendo estes calculados de forma a que o resultado seja o mais parecido possível com o obtido com a variante do método ELECTRE aqui proposta. O segundo é baseado em pesos diferentes para cada alternativa, calculados de forma otimista e equivale a um modelo DEA de input constante e de outputs iguais aos critérios considerados.

\subsection{Método aditivo de pesos iguais para as alternativas}

Este método calcula uma função de valor para cada alternativa, obtida por meio da soma ponderada dos valores dos critérios. Os pesos foram calculados de forma a que o resultado da soma ponderada fosse o mais próximo possível da alocação de classes obtida anteriormente. Entende-se como tal, uma divisão em que a diferença entre duas alternativas de classes diferentes (sendo as classes as mesmas obtidas anteriormente) seja sempre maior que a diferença entre duas alternativas da mesma classe.

Para tal, foi elaborado um Problema de Programação Linear (PPL) do tipo MinMax, no qual o objetivo é minimizar a maior diferença de valor entre duas alternativas da mesma classe. Existem as restrições adicionais de que aeroportos de uma classe superior devem ter uma 
avaliação não inferior a aeroportos de classes abaixo. Não foi feita restrição para aeroportos de uma mesma classe. O PPL é apresentado já na forma linearizada em (I), onde D, A, P e C são as variáveis de decisão referentes a, respectivamente, maior distância entre alternativas de uma mesma classe, peso para movimentação de aeronaves, peso para movimentação de passageiros e peso para movimentação de carga. Em (I) as linhas iniciadas em "!" representam linhas de comentário.

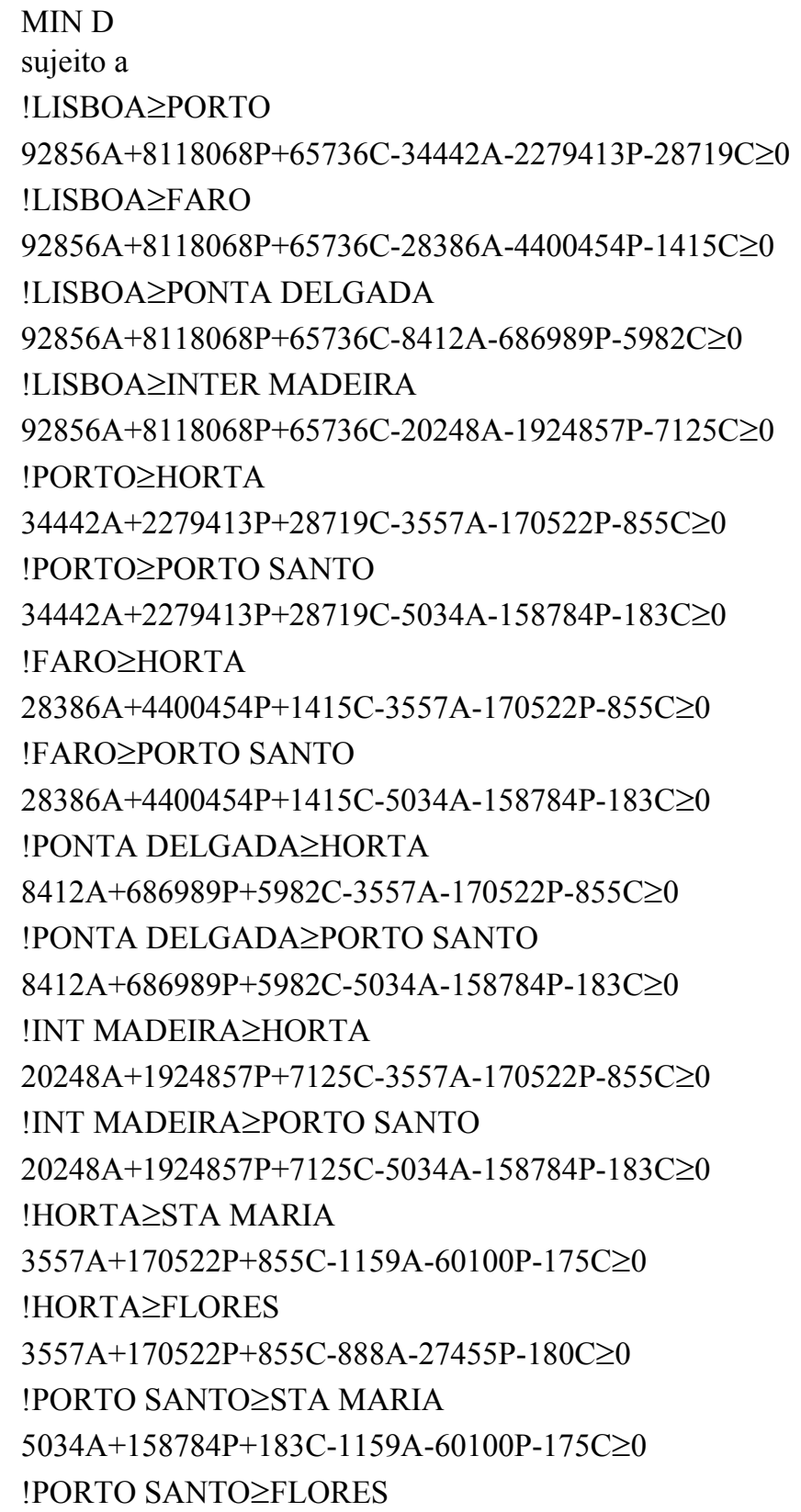




$$
\begin{aligned}
& 5034 \mathrm{~A}+158784 \mathrm{P}+183 \mathrm{C}-888 \mathrm{~A}-27455 \mathrm{P}-180 \mathrm{C} \geq 0 \\
& \mathrm{~A}+\mathrm{P}+\mathrm{C}=100000 \\
& \text { D-34442A-2279413P-28719C }+28386 \mathrm{~A}+4400454 \mathrm{P}+1415 \mathrm{C} \geq 0 \\
& \mathrm{D}+34442 \mathrm{~A}+2279413 \mathrm{P}+28719 \mathrm{C}-28386 \mathrm{~A}-4400454 \mathrm{P}-1415 \mathrm{C} \geq 0 \\
& \text { D-34442A-2279413P-28719C+20248A }+1924857 \mathrm{P}+7125 \mathrm{C} \geq 0 \\
& \mathrm{D}+34442 \mathrm{~A}+2279413 \mathrm{P}+28719 \mathrm{C}-20248 \mathrm{~A}-1924857 \mathrm{P}-7125 \mathrm{C} \geq 0 \\
& \text { D-34442A-2279413P-28719C+8412A }+686989 \mathrm{P}+5982 \mathrm{C} \geq 0 \\
& \mathrm{D}+34442 \mathrm{~A}+2279413 \mathrm{P}+28719 \mathrm{C}-8412 \mathrm{~A}-686989 \mathrm{P}-5982 \mathrm{C} \geq 0 \\
& \mathrm{D}+28386 \mathrm{~A}+4400454 \mathrm{P}+1415 \mathrm{C}-20248 \mathrm{~A}-1924857 \mathrm{P}-7125 \mathrm{C} \geq 0 \\
& \text { D-28386A-4400454P-1415C+20248A+1924857P+7125C } \geq 0 \\
& \mathrm{D}+28386 \mathrm{~A}+4400454 \mathrm{P}+1415 \mathrm{C}-8412 \mathrm{~A}-686989 \mathrm{P}-5982 \mathrm{C} \geq 0 \\
& \text { D-28386A-4400454P-1415C+8412A }+686989 \mathrm{P}+5982 \mathrm{C} \geq 0 \\
& \mathrm{D}+20248 \mathrm{~A}+1924857 \mathrm{P}+7125 \mathrm{C}-8412 \mathrm{~A}-686989 \mathrm{P}-5982 \mathrm{C} \geq 0 \\
& \text { D-20248A-1924857P-7125C }+8412 \mathrm{~A}+686989 \mathrm{P}+5982 \mathrm{C} \geq 0 \\
& \mathrm{D}+3557 \mathrm{~A}+170522 \mathrm{P}+855 \mathrm{C}-5034 \mathrm{~A}-158784 \mathrm{P}-183 \mathrm{C} \geq 0 \\
& \text { D-3557A-170522P-855C }+5034 \mathrm{~A}+158784 \mathrm{P}+183 \mathrm{C} \geq 0 \\
& \mathrm{D}+1159 \mathrm{~A}+60100 \mathrm{P}+175 \mathrm{C}-888 \mathrm{~A}-27455 \mathrm{P}-180 \mathrm{C} \geq 0 \\
& \text { D-1159A-60100P-175C+888A }+27455 \mathrm{P}+180 \mathrm{C} \geq 0 \\
& \mathrm{D}, \mathrm{A}, \mathrm{P}, \mathrm{C} \geq 0
\end{aligned}
$$

Os pesos obtidos como resultado do PPL (I) são 0,$186097 ; 0,000000 ; 0,813903$ para movimentação de aeronaves, passageiros e carga, respectivamente. A Tabela 5 apresenta a ordenação e o resultado da soma ponderada com estes pesos.

Tabela 5 - Resultados da soma ponderada com pesos obtidos de (I).

\begin{tabular}{cc}
\hline Alternativas & Soma ponderada \\
\hline Lisboa & $70.782,9$ \\
Porto & $29.784,0$ \\
Internacional da Madeira & $9.567,2$ \\
Faro & $6.434,2$ \\
Ponta Delgada & $6.434,2$ \\
Horta & $1.357,8$ \\
Porto Santo & $1.085,8$ \\
Santa Maria & 358,1 \\
Flores & 311,8 \\
\hline
\end{tabular}

Verifica-se que é possível encontrar a mesma divisão em classes anterior, desde que os limites sejam apropriadamente escolhidos. Não surpreende que, neste caso, os inconvenientes da soma ponderada para a alocação em classes mencionados anteriormente 
não se façam notar, já que os pesos foram calculados com esse objetivo. Por outro lado, um dos critérios teve peso nulo, o que significa que ele não é levado em conta na ordenação e, portanto, este modelo viola o axioma da exaustividade de Roy.

\subsection{Modelo com vetor de pesos diferente para cada alternativa}

Este modelo consiste em calcular os pesos usados na soma ponderada de cada alternativa de modo a maximizar o valor obtido para cada uma com as restrições de que o vetor de pesos de uma alternativa aplicado às demais não pode resultar em valor superior a 1 (um). Formalmente, para cada alternativa é rodado o PPL apresentado em (II), onde $u_{P i}, u_{C i}$ e $u_{A i}$ são, respectivamente, os pesos atribuídos à movimentação de passageiros, de carga e de aeronaves pela alternativa $i$ em avaliação; $P_{j}, C_{j}$ e $A_{j}$ são os valores de movimentação de passageiros, de carga e de aeronaves da alternativa $j$.

$$
\begin{aligned}
& \max \left(u_{P i} P_{i}+u_{C i} C_{i}+u_{A i} A_{i}\right) \\
& \text { sujeito a } \\
& u_{P i} P_{j}+u_{C i} C_{j}+u_{A i} A_{j} \leq 1, \forall j \\
& u_{P i}, u_{C i}, u_{A i} \geq 0
\end{aligned}
$$

Este é um modelo DEA com retornos constantes de escala (Charnes et al., 1978) em que o input é constante e unitário, como mostrado em Soares de Mello \& Gomes (2004).

Ao interpretar-se DEA como uma ferramenta multicritério (Farinaccio \& Ostanello, 1999; Belton \& Stewart, 1997), DEA resolve a problemática P $\gamma$. Barr et al. (2000) e Tavares (1998) propõem um artifício para DEA estabelecer uma classificação, que consiste em estabelecer camadas de eficiência. Essas camadas são obtidas da seguinte forma (Gomes et al., 2003): as alternativas com $100 \%$ de eficiência formam a camada 1. Essas alternativas são, então, retiradas do conjunto de análise e roda-se novamente o modelo DEA. As alternativas eficientes neste subconjunto formam a camada 2. O processo repete-se até que todas as alternativas tenham sido retiradas do conjunto inicial. Repare-se que esta também é uma divisão em classes que não exige definição de parâmetros por parte do decisor. As próprias alternativas estabelecem a divisão em classes, similarmente à variante do ELECTRE I proposta neste artigo.

Ao aplicar-se o modelo DEA em camadas, usando-se o software SIAD (Angulo Meza et al., 2004), obteve-se o conjunto de camadas mostrado na Tabela 6.

Tabela 6 - Camadas do modelo DEA.

\begin{tabular}{cc}
\hline Camadas & Alternativas \\
\hline I & Lisboa \\
II & Porto, Faro \\
III & Internacional da Madeira \\
IV & Ponta Delgada \\
V & Horta, Porto Santo \\
VI & Flores, Santa Maria \\
\hline
\end{tabular}


Verifica-se uma grande semelhança com a alocação obtida pela variante do método ELECTRE. A única diferença é que o modelo DEA em camadas subdividiu a classe II original. Dessa forma, o modelo DEA em camadas produziu um resultado intermediário entre a ordenação obtida quando os decisores estavam certos quanto aos seus julgamentos (Tabela 3) e a divisão em classes resultante quando essas certezas foram relaxadas (Tabela 4).

\section{Conclusões}

Este artigo mostrou como é possível tirar partido da aparente desvantagem do método ELECTRE de criar freqüentemente ciclos de intransitividade. Estes ciclos, quando existentes, impedem uma ordenação total e podem gerar problemas na solução da P $\alpha$, o que obriga a solicitar informação adicional ao decisor. Já para a $\mathrm{P} \beta$ os ciclos mostraram-se úteis pois, por meio de relações de pseudo-equivalência, geram as classes pretendidas.

A primeira configuração usada conduziu a uma ordenação total dos aeroportos portugueses em termos de movimentação. Aparentemente há uma grande diferença entre esses aeroportos e uma grande semelhança nas classificações individuais em cada critério.

No entanto, a segunda configuração, ao relaxar as certezas do decisor, mostra a ocorrência de alguns ciclos no grafo. Fora desses ciclos está apenas o aeroporto de Lisboa, maior aeroporto português quaisquer que sejam os métodos ou critérios utilizados. No entanto, nas demais classes surgem ciclos. O primeiro contém os aeroportos de Porto e Faro, situados no continente e porta de entrada das regiões Norte e Algarve, respectivamente, e o aeroporto mais movimentado de cada arquipélago (Internacional da Madeira e Ponta Delgada). A classe seguinte é formada pelos aeroportos secundários dos Açores e da Madeira, e a última classe é constituída apenas de pequenos aeroportos dos Açores.

A classe formada pelos aeroportos de Porto, Faro, Ponta Delgada e Internacional da Madeira apresenta uma característica interessante: são aeroportos que servem de principal porta de entrada para regiões fortemente turísticas. Dois deles, localizados em ilhas, não têm (como é evidente) acesso rodoviário ao continente europeu, região geradora do principal tráfego turístico. Já o de Faro, fica no extremo sul de Portugal (no Algarve) e, há até bem pouco tempo, tinha acessos rodoviários bastante precários. Assim, estes três aeroportos, além de estarem em uma mesma classe por questões de tamanho, ainda apresentam, com exceção do aeroporto do Porto, características geográficas de acesso com alguma semelhança.

O emprego do método de soma ponderada mostrou-se de pouca utilidade prática. Mostrou-se que era possível obter resultados semelhantes, mas que somente foram conseguidos por meio da informação "final", ou seja, conhecendo-se a alocação já feita pelo método ELECTRE. Ainda assim, o vetor de pesos não representa opinião do decisor e ao atribuir peso nulo a um dos critérios violou o axioma da exaustividade.

Já o segundo método de comparação (modelo DEA em camadas) apresentou resultados bastante semelhantes àqueles obtidos inicialmente. Teve como desvantagem a geração de um número maior de classes e como vantagem não necessitar de hipóteses de relaxação ou não de certezas do decisor. Ressalta-se ainda que a semelhança de resultados coincide com a semelhança de filosofia: tanto no modelo DEA quanto na variante do método ELECTRE I proposta neste artigo as classes, quando existem, são determinadas pelas próprias alternativas, sem a interferência do decisor. 
Neste artigo, um dos autores, com razoável conhecimento de aeroportos e transporte aéreo, atuou como decisor. Em uma situação real, uma autoridade aeroportuária ficaria responsável pela elicitação de seus próprios valores, através de uma ausculta, pelo método apresentado neste artigo. Dessa forma, o método proposto foi validado por meio de um experimento, no qual um dos autores atuou como agente de decisão e, por mostrar-se válido, sugere-se sua utilização em situações com outros decisores.

A aplicação aos aeroportos brasileiros do método variante do ELECTRE I apresentado neste artigo pode revelar classes interessantes, mas não há acesso fácil aos dados completos necessários para usar o método tal como foi desenvolvido. Pretende-se, em trabalhos futuros, fazer uma adaptação do método para poder trabalhar com a ausência de alguns dados. O método ELECTRE Is poderá revelar-se útil para este fim.

\section{Agradecimentos}

Ao CNPq pelo apoio financeiro, processo 301095/2003-5.

\section{Referências Bibliográficas}

(1) ANA (2002). ANA Aeroportos de Portugal AS. Disponível em: $<$ http://www.ana-aeroportos.pt $>$. Acesso em: 15 out. 2002.

(2) Angulo Meza, L.; Biondi Neto, L.; Soares de Mello, J.C.C.B.; Gomes, E.G. \& Coelho, P.H.G. (2004). FSDA - Free Software for Decision Analysis (SLAD - Software Livre de Apoio à Decisão): A software package for Data Envelopment Analysis models. Memórias del XII Congreso Latino-Iberoamericano de Investigación de Operaciones y Sistemas - CLAIO, La Habana, Cuba.

(3) Bana e Costa, C.A.; Ensslin, L.; Corrêa, E.C. \& Vansnick, J.C. (2002). Decision support systems in action: Integrated application in a multicriteria decision aid process. European Journal of Operational Research, 113(2), 315-335.

(4) Bana e Costa, C.A. \& Vansnick, J.C. (1994). MACBETH: An interactive path towards the construction of cardinal value functions. International Transactions in Operational Research, 1(4), 489-500.

(5) Barba-Romero, S. \& Pomerol, J.C. (1997). Decisiones Multicriterio: Fundamentos Teóricos y Utilización Práctica, Colección de Economía. Universidad de Alcalá, Espanha.

(6) Barr, R.S.; Durchholz, M.L. \& Seiford, L. (2000). Peeling the DEA onion: Layering and rank-ordering DMUs using tiered DEA. Technical Report, Southern Methodist University.

(7) Belton, V. \& Stewart, T.J. (1997). DEA and MCDA: competing or complementary approaches? Working Paper, University of Strathclyde, Scotland.

(8) Boaventura Netto, P.O. (2002). Grafos: Teoria, Modelos, Algoritmos. 2a . ed., Edgard Blucher, São Paulo.

(9) Bouyssou, B. (1990). Building criteria: a prerequisite for MCDA. In: Readings in Multiple Criteria Decision Aid [edited by C.A. Bana e Costa], Springer Verlag, Berlin. 
(10) Charnes, A.; Cooper, W.W. \& Rhodes, E. (1978). Measuring the efficiency of decisionmaking units. European Journal of Operational Research, 2, 429-444.

(11) Costa, H.G.; Soares, A.C. \& Oliveira, P. (2004). Escolha de prestadoras de serviço para transporte de materiais perigosos: abordagem multicritério pelo ELECTRE TRI. Gestão \& Produção, 11(2), 1-15.

(12) Dias, L.C. (2002). Apontamentos de análise de decisão: como considerar múltiplos critérios. Faculdade de Economia, Universidade de Coimbra, Coimbra, $95 \mathrm{p}$.

(13) Dias, L.M.C.; Almeida, L.M.A.T. \& Clímaco, J.C.N. (1996). Apoio Multicritério à Decisão. Faculdade de Economia, Universidade de Coimbra, Coimbra.

(14) Domingues, H.H. \& Iezzi, G. (1976). Introdução à Álgebra. Atual Editora Ltda., São Paulo.

(15) Espírito Santo Jr., R.A. (2000). Concentração no transporte aéreo e os possíveis impactos sobre os consumidores, a sociedade e a economia. Transporte em Transformação $V, 155-170$.

(16) Farinaccio, F. \& Ostanello, A. (1999). Evaluation of DEA validity as a MCDA/M tool: some problems and issues. Technical Report TR-99-06, Dipartimento de Informatica, Università di Pisa, Itália.

(17) Fernandes, E. \& Pacheco, R.R. (2002). Efficient use of airport capacity. Transportation Research Part A: Policy and Practice, 36(3), 225-238.

(18) Gomes, E.G.; Soares de Mello, J.C.C.B. \& Estellita Lins, M.P. (2003). Busca seqüencial de alvos intermediários em modelos DEA com soma de outputs constante. Investigação Operacional, 23(2), 163-178.

(19) Gomes, L.F.A.M. \& Rocha, M.A.L. (1999). Análise multicritério pelos métodos ELECTRE III e ELECTRE IV - Um lançamento de um novo serviço dos correios. Engevista, 2(4), 5-14.

(20) Gomes, L.F.A.M.; Gomes, C.F.S. \& Almeida, A.T. (2002). Tomada de Decisão Gerencial Enfoque Multicritério. Ed. Atlas, São Paulo.

(21) Gomes, L.F.A.M.; Gonzalez Araya, M.C. \& Carignano, C. (2004). Tomada de Decisões em Cenários Complexos. Ed. Thompson, São Paulo.

(22) Miranda, C.M.G. \& Almeida, A.T. (2003). Avaliação de Pós-Graduação com Método ELECTRE TRI - O Caso de Engenharias III da CAPES. Revista Produção, 13(3), 101-112.

(23) Miranda, C.M.G. \& Almeida, A.T. (2004). Visão Multicritério da Avaliação de Programas de Pós-Graduação pela CAPES - O Caso da Área Engenharias III Baseado nos Métodos ELECTRE II e MAUT. Gestão \& Produção, 11(2), 1-20.

(24) Palhares, G.L. (2001). Transporte Aéreo e Turismo: gerando desenvolvimento socioeconômico. Ed. Aleph, São Paulo.

(25) Rogers, M.; Bruen, M. \& Maystre, L.Y. (2000). Electre and Decision Support: Methods and Application in Engineering and Infrastructure Investment. Kluwer Academic Publishers, Boston. 
(26) Roy, B. \& Bertier, P. (1971). La méthode ELECTRE II. SEMA (Metra International) Note de Travail, 142.

(27) Roy, B. \& Bertier, P. (1973). La méthode ELECTRE II - Une application au média-planning. In: $O R^{\prime} 72$ [edited by M. Ross], North-Holland Publishing Company, 291-302.

(28) Roy, B. \& Bouyssou, D. (1993). Aide multicritère à la décision: méthods et cas. Ed. Economica, Paris.

(29) Roy, B. (1968). Classement et choix en présence de points de vue multiples (la méthode ELECTRE). RIRO, 8, 57-75.

(30) Roy, B. (1985). Méthodologie multicritère d'Aide a la dècision. Paris. Economica.

(31) Soares de Mello, J.C.C.B. \& Gomes, E.G. (2004). Eficiências Aeroportuárias: Uma Abordagem Comparativa com Análise de Envoltória de Dados. Revista de Economia e Administração, 3(1), 15-23.

(32) Soares de Mello, J.C.C.B.; Gomes, E.G.; Gomes, L.F.A.M.; Biondi Neto, L. \& Leta, F.R. (2003b). Seleção de rota aérea com o uso do apoio multicritério à decisão. Engevista, 5(10), 71-84.

(33) Soares de Mello, J.C.C.B.; Gomes, E.G.; Leta F.R. \& Vidal Pessolani, R.B. (2003a). Conceitos básicos do Apoio Multicritério à Decisão e sua aplicação no projeto aerodesign. Engevista, 5(8), 22-35.

(34) Soares de Mello, J.C.C.B.; Gomes, L.F.A.M.; Gomes, E.G. \& Soares de Mello, M.H.C. (2003c). Gestão dos desportos: uma aplicação de métodos ordinais à Fórmula 1. Revista Portuguesa e Brasileira de Gestão, 2(3), 79-87.

(35) Tavares, G. (1998). Data envelopment analysis: the basic models and their main extensions. A model to analyse the modernisation of telecommunication services in OECD countries. Tese de Mestrado, Faculdade de Ciências e Tecnologia, Universidade de Coimbra, Portugal.

(36) Yu, W. \& Roy, B. (1992). Aspects mèthodologiques et manuel d'utilisation. Document N. 74, Université de Paris Dauphine. Paris. 